\title{
Prediction and Derivation of the Hubble Constant from Subatomic Data Utilizing the Harmonic Neutron Hypothesis
}

\author{
Donald William Chakeres ${ }^{1}$, Richard Vento ${ }^{2}$ \\ ${ }^{1}$ Department of Radiology, The Ohio State University, Columbus, USA \\ ${ }^{2}$ Columbus State Community College, Columbus, USA \\ Email: donald.chakeres@osumc.edu, rpvento@aol.com
}

Received 5 February 2015; accepted 23 February 2015; published 27 February 2015

Copyright (C) 2015 by authors and Scientific Research Publishing Inc.

This work is licensed under the Creative Commons Attribution International License (CC BY).

http://creativecommons.org/licenses/by/4.0/

(c) (i) Open Access

\section{Abstract}

Purpose: To accurately derive $H_{0}$ from subatomic constants in abscence of any standard astronomy data. Methods: Recent astronomical data have determined a value of Hubble's constant to range from 76.9+3.9 ${ }_{-3.4^{+10.0}}{ }_{-8.0}$ to $67.80 \pm 0.77(\mathrm{~km} / \mathrm{s}) / \mathrm{Mpc}$. An innovative prediction of $H_{0}$ is obtained from harmonic properties of the frequency equivalents of neutron, $n^{0}$, in conjunction with the electron, e; the Bohr radius, $\alpha_{0}$; and the Rydberg constant, $R$. These represent integer natural unit sets. The neutron is converted from its frequency equivalent to a dimensionless constant, $\left(v_{n^{0}}\right) s=\left(m_{n}^{0} c^{2}\right) \mathrm{s} / \mathrm{h}=2.27185907(11) \times 10^{23} \mathrm{~Hz}-\mathrm{s}$, where " $\mathrm{h}$ " = Planck's constant, and "s" is measured in seconds. The fundamental frequency, $V_{f}$, is the first integer series set $\left(v_{n^{0}}\right) s=V_{f}=\left\{v_{f}\right\}$. All other atomic data are scaled to $V_{f}$ as elements in a large, but a countable point set. The present value of $H_{0}$ is derived and $\Omega_{M}$ assumed to be 0 . An accurate derivation of $H_{0}$ is made using a unified power law. The integer set of the first twelve integers $N_{12}\{1,2, \cdots, 11,12\}$, and their harmonic fractions $N_{12}^{\prime}\{1 / 1,1 / 2, \cdots, 1 / 11,1 / 12\}$ exponents of $V_{f}$ represent the first generation of bosons and particles. Thepartial harmonic fraction, $-3 / 4$, is exponent of $V_{f}$ which represents $H_{0}$. The partial fraction $3 / 4$ is associated with a component of neutron beta decay kinetic energy. Results: $H_{0}$ is predicted utilizing a previously published line used to derive Planck time, $t_{p}$. The power law line of the experimental $H_{0}$ and $t_{p}$ conforms to the predicted line. Conclusions: $H_{0}$ can be predicted from subatomic data related to the neutron and hydrogen.

\section{Keywords}

Hubble Constant, Neutron, Unification Model, Planck Time, Quantum Gravity, Neutron Beta Decay, Neutrino 


\section{Introduction}

\subsection{The Hubble Constant}

Hubble's law refers to the observation that objects at greater than 10 megaparsecs have a Doppler shift interpretable as a relative velocity. The Doppler shift is most commonly quoted as a velocity in $(\mathrm{km} / \mathrm{s}) / \mathrm{Mpc}$. Galaxies appear to be moving at a rate proportional to their distance from the Earth. This is typically interpreted as evidence of the expansion of the Universe. A high precision Hubble constant $H_{0}$, is an important physical constant, [1]-[6]. Hubble's Law relates a velocity to $H_{0}$ as a proportionality constant with units of $\mathrm{s}^{-1}$ times the proper distance, D. The reciprocal of $H_{0}$ is the Hubble time. The reported velocities at one Mpc vary with the model and published values include: $76.9^{+3.9}{ }_{-3.4^{+10.0}}^{+8.0} \mathrm{~km} \cdot \mathrm{s}^{-1} \cdot \mathrm{Mpc}^{-1}, 69.32 \pm 0.80 \mathrm{~km} \cdot \mathrm{s}^{-1} \cdot \mathrm{Mpc}^{-1}, 74.3 \pm 2.1$ $\mathrm{km} \cdot \mathrm{s}^{-1} \cdot \mathrm{Mpc}^{-1}, 67.3 \pm 1.2 \mathrm{~km} \cdot \mathrm{s}^{-1} \cdot \mathrm{Mpc}^{-1}$, Table 1, [1]-[6]. The methods, probes, of measurement and derivation of the $H_{0}$ are different. This leads to divergent estimated values based on the methods and model. The methods include the Hubble telescope, Chandra and Sunyaev-Zeldovich Effect data from the Owens Valley Radio Observatory and the Berkeley-Illinois-Maryland Association interferometric arrays, Wilkinson Microwave Anisotropy Probe, and cosmic microwave background, CMB, temperature and lensing-potential power spectra The experimental Hubble rates range from 2.18(4) $\times 10^{-18} \mathrm{~s}^{-1}$ to $2.49(12) \times 10^{-18} \mathrm{~s}^{-1}$. The reported Hubble time, $H_{0}^{-1}$, equals approximately $4.35 \times 10^{17} \mathrm{~s}$ or $13.8 \mathrm{byr}$. The approximate Hubble length equals $c \times H_{0}^{-1}$ or 13.8 blyr. In this model the predicted $H_{0}$ is assumed to be present experimental value with an $\Omega_{M}$ of 0 .

\subsection{The Goals}

The goal of this work is to derive a high precision $H_{0}$, and subsequently a Hubble length, and Hubble time from natural unit frequency equivalents as integer sets of the neutron, $n^{0}, v_{n^{0}}, V_{f}$; the hydrogen ionization energy; the Rydberg constant $R, v_{R}$; the Bohr radius $\alpha_{0}, v_{\alpha_{0}}$; and the electron, $e, v_{e}$; and the finite integer and har-

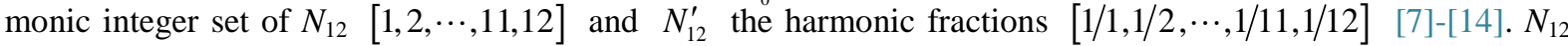
are referred to as integer fraction exponents, $n_{\text {ife }}$. Another goal is to demonstrate that experimental Planck time squared, $t_{p}^{2}$ and $H_{0}$ follow a previously predicted power law relationship used to derive $t_{p}^{2}$. [9] The proportionality constants of $t_{p}^{2}$, and $H_{0}$ ratios with $v_{n^{0}}$ are derived, and compared to the known experimental values.

\subsection{The Harmonic Neutron Hypothesis ( $\mathrm{HNH})$}

The following is a review and explanation of the harmonic neutron hypothesis. It was initially copyrighted in 2006 and published in 2009, [7]-[14]. All of the physical constants are evaluated as frequency equivalents, and secondarily as dimensionless coupling constant ratios in an exponential integer or integer fraction system. Any single physical unit could be utilized, but Hertzian frequency $(\mathrm{Hz})$ was arbitrarily chosen since the whole physical

Table 1. Comparison of experimental, experimental line fit, and derived $H_{0}$, velocities, exponents, and $\delta s$ for $H_{0}$ and $t_{p}^{2}$. Table 1 lists s ${ }^{-1}$, and $\mathrm{s}^{2}$ for the four experimental known, line fit known, and derived values for $H_{0}$, and $t_{p}^{2}$. The derived and line fit known experimental data are remarkable similar.

\begin{tabular}{|c|c|c|c|c|}
\hline & $\mathrm{s}^{2}$ or $\mathrm{s}^{-1}$ & $\mathrm{~km} \cdot \mathrm{s}^{-1} \cdot \mathrm{Mpc}^{-1}$ & Exponent & $\delta$ \\
\hline$H_{0 k}$ & $2.49(12) \times 10^{-18} \mathrm{~s}^{-1}$ & $76.9^{+3.9}-3.4^{+10.0}-8.0$ & $-7.53(12) \times 10^{-1}$ & $-3.70(90) \times 10^{-3}$ \\
\hline$H_{0 k}$ & $2.408(67) \times 10^{-18} \mathrm{~s}^{-1}$ & $74.3 \pm 2.1$ & $-7.543(8) \times 10^{-1}$ & $-4.33(50) \times 10^{-3}$ \\
\hline$H_{0 k}$ & $2.24(2) \times 10^{-18} \mathrm{~s}^{-1}$ & $69.32 \pm 0.80$ & $-7.556(16) \times 10^{-1}$ & $-5.62(20) \times 10^{-3}$ \\
\hline$H_{0 k}$ & $2.18(4) \times 10^{-18} \mathrm{~s}^{-1}$ & $67.3 \pm 1.2$ & $-7.562(3) \times 10^{-1}$ & $-6.17(32) \times 10^{-3}$ \\
\hline$H_{0 k l i n e ~ f i t}$ & $2.33(11) \times 10^{-18} \mathrm{~s}^{-1}$ & $71.9(36)$ & $-7.549(11) \times 10^{-1}$ & $-4.99(32) \times 10^{-3}$ \\
\hline$H_{0 d}$ & $2.29726680(12) \times 10^{-18} \mathrm{~s}^{-1}$ & $70.886246(4)$ & $-7.55202112(1) \times 10^{-1}$ & $-5.2021124(11) \times 10^{-3}$ \\
\hline$t_{P k}^{2}$ & $1.82611(11) \times 10^{-86} \mathrm{~s}^{2}$ & & $-3.670879(12)$ & $-1.37371(1) \times 10^{-2}$ \\
\hline$t_{P k \text { line fit }}^{2}$ & $1.82611(11) \times 10^{-86} \mathrm{~s}^{2}$ & & $-3.670879(12)$ & $-1.37371(1) \times 10^{-2}$ \\
\hline$t_{P d}^{2}$ & $1.8261712(1) \times 10^{-86} \mathrm{~s}$ & & $-3.670879366(1)$ & $-1.3736509(1) \times 10^{-2}$ \\
\hline
\end{tabular}


system can logically be evaluated as a unified quantum spectrum. The primary natural unit is $V_{f}$. All possible physical values are defined by $V_{f}$ and integer fractions. The unit system of the HNH is maximally simplified with the units for Planck's constant, charge, and speed of light all equaling 1. Energy, mass, frequency, temperature are all equal. The distance is 1 divided by frequency. The speed of light equals $v_{n^{0}}$ times the distance $1 /\left(v_{n^{0}} s\right)$, Compton radius of the neutron. The maximum Lorentz factor equals $\left(v_{n^{0}} s\right)$. Velocity squared is $n /\left(v_{n^{0}} s\right)^{2}$.

The primary hypothesis is that the fundamental constants are inter-related by simple, ubiquitous mathematical and geometric integer patterns. The first twelve integers, $N_{12}$, and their harmonic fractions, $N_{12}^{\prime}$, provide the basis for a coherent theory defining the first generation of bosons and particles, used in a power law computation for defining physical phenomena, Table 2. The model utilizes the dimensional analysis methods of Rayleigh's method and the Buckingham Pi Theorem, where the exponential base is the dimensionless neutron annihilation frequency, Hz-s, $\left(v_{n^{0}} s\right), V_{f}$, and the exponents are combinations of 1 plus or minus $N_{12}^{\prime}$. Buckingham's pi theorem states that physical laws are independent of the form of the physical units. Therefore, acceptable laws of physics are homogeneous in all dimensions.

The Equality Pair Transformations (EPTs) inter-relate matter, electromagnetic energy, and kinetic energy transformations. EPT are common physical phenomena that can be described by Feynman diagrams, but necessitate a definitional approach when utilized in this model. Each EPT is associated with a point transformation from one state to another, or from one force to another, such as, kinetic energy to electromagnetic energy, electromagnetic energy to matter, or vice-versa. This occurs when there is a scale equality of two different states or forces. The pair is identically scaled phenomena, but can represent two different dual (paired) physical manifestations of different forces or states. This is the essence of particle-wave duality paradox. Examples are matter-antimatter pair production or annihilation; or the transformation of electromagnetic energy to kinetic energy as in the photoelectric effect. Not only is there a conservation-equality of total energy-matter, but also a transformation of state or force. These transformations are always associated with symmetric pairs.

Table 2. First generation particles and bosons. Table 2 lists the first generation physical constants related to neutron beta decay, $n_{i e}, n_{i f e}$, and the partial harmonic fractions associated with $N_{12}, N_{12}^{\prime}$. There is no physical entity associated with the $n_{\text {ife }}, 9$.

\begin{tabular}{|c|c|c|}
\hline Constant unit & $n_{\text {ie }}$ or $n_{\text {ife }}$ & $1 \pm 1 / n_{\text {ife }}, q f$ \\
\hline Elemental gravitational kinetic energy of the electron in hydrogen & -1 & -1 \\
\hline h, electromagnetic energy, boson & 0 & $0,1-1 / 1$ \\
\hline$n^{0}$, elemental mass, strong force & 1 & 1 \\
\hline $\begin{array}{l}\text { Beta decay kinetic energy, anti-neutrino mass, cosmic background microwave, } \\
\text { CMB, peak spectral radiance }\end{array}$ & 2 & $1 / 2,1-1 / 2$ \\
\hline Rydberg constant, $R$, em energy, boson & 3 & $2 / 3,1-1 / 3$ \\
\hline Beta decay kinetic energy, muon anti-neutrino & 4 & $3 / 4,1-1 / 4$ \\
\hline Bohr radius, $\alpha_{0}$, distance, or beta decay kinetic energy & 5 & $4 / 5,1-1 / 5$ \\
\hline Beta decay kinetic energy, Tau anti-neutrino & 6 & $5 / 6,1-1 / 6$ \\
\hline Electron, $e$, mass, matter & 7 & $6 / 7,1-1 / 7$ \\
\hline Beta decay kinetic energy & 8 & $7 / 8,1-1 / 8$ \\
\hline Up quark, $u$, matter & 10 & $9 / 10,1-1 / 10$ \\
\hline Down quark, $d$, matter & 11 & $10 / 11,1-1 / 11$ \\
\hline$\alpha^{-1}$, reciprocal fine structure constant, coupling constant & 11 & $1 / 11$ \\
\hline Higgs boson, $H^{0}$, boson & 11 & $12 / 11,1+1 / 11$ \\
\hline W, Z, boson & 12 & $13 / 12,1+1 / 12$ \\
\hline
\end{tabular}


The primary fundamental EPT scaling HNH is neutron anti-neutron pair production, and is the scaling factor used to derive further observable phenomena. The fundamental EPT ratio set is composed of a natural physical unit as a consecutive integer series representing the transformation of electromagnetic energy into frequency multiples matter associated with neutron/anti-neutron pair production. The integrally spaced dimensionless elements, $v_{f}$ in $V_{f}$, are based on the ratio of the respective annihilation frequencies of that physical constant to that of the neutron. At the point where the photon integer frequency series has enough energy to be scaled identically with elemental neutral matter equivalent represents the fundamental EPT. The series restarts again at 1 with each integer representing the number of nucleons in elemental matter or groups of nucleons at the EPT of pair production point. Elements in the set $V_{f}$ scale all of the possible physical phenomena under consideration.

\subsection{The Empirical Observations Leading to the $\mathrm{HNH}$}

The neutron annihilation frequency, $\left(v_{n^{0}} s\right)$, is the fundamental coupling constant, and the basis for EPTs on the set $V_{f}$. We assume that there exists a gravitational binding energy of the electron to the proton in hydrogen, equally as important to the units of gravitational energy, as the ionization energy of hydrogen is to the electromagnetic force. The genesis of this hypothesis was based on the empirical observation that there are integer exponent relationships of $v_{n^{0}} s$ between the twice the frequency equivalent of the gravitational binding energy of the electron in hydrogen, the energy of Planck's constant, h-Hz, and the frequency equivalent of the neutron.

Twice the frequency equivalent of the gravitational binding energy of the electron in hydrogen, $2 \times$ $2.90024(22) \times 10^{-24} \mathrm{~Hz}$ equals $5.80048(44) \times 10^{-24} \mathrm{~Hz}$. We label here the gravitational binding energy of the electron in hydrogen as the elemental graviton. The frequency equivalent of Planck's constant, h, is $1 \mathrm{~Hz}$. Here, $\left(v_{n^{0}}\right) \mathrm{Hz}$ is $2.271859078(50) \times 10^{23} \mathrm{~Hz}$. The reciprocal of $\left(v_{n^{0}} s\right)$ is $4.4016815(1) \times 10^{-24}$, and is almost identical to twice the binding gravitational frequency of the electron in hydrogen.

The factor two in the gravitational binding energy of the electron to the proton arises from the fact that it is a kinetic energy. This "2" has the same origin as the "2" in the Schwarzschild radius equation, $r_{\text {sch }}=2 \mathrm{GM} / \mathrm{c}^{2}$, and both transform kinetic energy into an electromagnetic equivalent. This is also the "2" associated with an equality "pair". All three force-state unit values are separated by a ratio of $\left(v_{n^{0}} s\right)$ Each represents unit step values for elemental gravitational kinetic, electromagnetic, and the elemental strong nuclear forces. The frequency equivalent of the gravitational electron binding energy when multiplied by $\left(v_{n^{0}} s\right)$ equals 1.3178, and the normalized value of $1 \mathrm{~Hz}$ for Planck's constant h, times $\left(v_{n^{0}} s\right)$, equals $v_{n^{0}}$ in Hz. The coupling constant ratios are represented by an injective mapping of the sequence: $\{-1,0,1,2\}$ to exponents of $\left[\left(v_{n^{0}} s\right)\right]^{n}$ Hz. These integer exponents, are referred to as $n_{\mathrm{ie}}$ for integer exponents. Each $n_{\mathrm{ie}}$ is associated with a different force and state. Thus there is a discrete quantum relationship similar to blackbody spectra, and a power law. The distribution of energy over time is exponential for many physical systems including magnetic resonance relaxation.

Energy multiples of Planck's constant, h, is also an integer based wavelength or frequency system. This is identical to the resonant modes of a vibrating string. Planck's constant represents integral units of electromagnetic energy. Though $\mathrm{h}$ is quantum by definition, its actual physical manifestation in black body radiation appears to be continuous. When the divisions between the physical values associated with each $\mathrm{n}$ unit are smaller than experimental accuracy then the physical system appears to be Almost Everywhere (A.E.) continuous, but is none the less conceptually and mathematically integer-based Figure 1.

If this initial EPT observation is valid, then there logically should be a similarly scaled transformation between the unit values of the gravitational and electromagnetic forces. At $\left(v_{n^{0}} s\right)$ integral steps of gravitational units, i.e. twice the elemental graviton, this energy could represent an electromagnetic wave with a frequency of $1 \mathrm{~Hz}$. At $\left(v_{n^{0}} s\right)$ integral steps of electromagnetic units, at $1 \mathrm{~Hz}$, this energy could represent a neutron, at a frequency of $v_{n^{0}}$ Hz. At $\left(v_{n^{0}} s\right)$ integral steps of nucleon units, $v_{n^{0}} \mathrm{~Hz}$, this mass could represent the unit mass of a black hole, at a frequency of $\left[\left(v_{n^{0}} s\right)\right]^{2} \mathrm{~Hz}, 5.16134367(12) \times 10^{46} \mathrm{~Hz}$. This is an extremely dense from of matter, and must be the mass of individual unit forming a black hole analogous of neutrons forming elemental matter. This latter mass's Compton radius is smaller than its Schwarzschild radius, and must represent the matter of a black hole. 

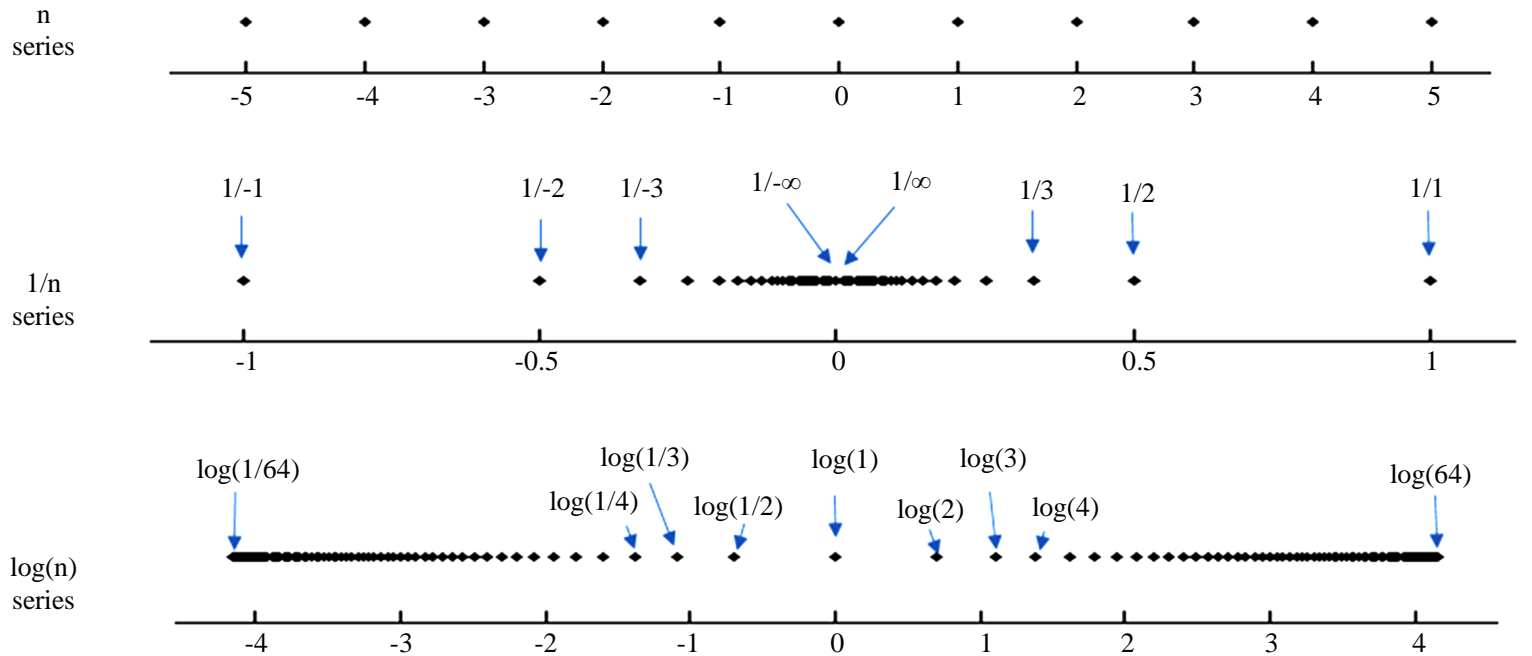

Figure 1. Point plots of a consecutive integer series in linear, $1 / n$, and $\log _{e}(n)$ formats. Figure 1 plots three different geometric, mathematical formats of the consecutive integer sequence of $n$ from $-\infty$ to $\infty$. All three of these are seen in the model, but each in the appropriate physical and mathematical context. All are discrete series of individual points. The top row is a standard linear plot which is bound on both extremes by $-\infty$ to $\infty$. The middle plot is $1 / n$. It is bound from -1 to +1 . This is the origin of the fundamental constants where there is dense clustering the closer the mass is to the neutron, and increasingly sparse at the extremes. Figure 2, Figure 3 demonstrates that a $2 \mathrm{~d}$ unit based system is associated with these identical harmonic fraction possibilities. The lower plot is $\log _{e}(n)$. It also extends from by $-\infty$ to $\infty$. This pattern also has a pseudo-continuous appearance as $n$ increases. For a large $V_{f}$ system only those $n$ values that are small will appear to be discrete.

\subsection{Assignment of Fundamental Physical Constants to Principal Quantum Numbers, $\boldsymbol{n}_{\text {ief }}$}

In this method all of the physical phenomena are evaluated as exponents of $\left[\left(v_{n^{0}} s\right)\right]$. Each physical constant is associated with an integer identical to all quantum systems. The known exponent, $\exp _{\mathrm{k}}$, of the base $\left[\left(v_{n^{0}} s\right)\right]$ for any physical constant is the natural $\log \left(\log _{\mathrm{e}}\right)$ of the frequency equivalent of that constant divided by $\log _{\mathrm{e}}\left[\left(v_{n^{0}} s\right)\right]$. This is a classic exponent base transformation. The difference between the known exponent and its associated partial fraction represents the $\delta$ factor.

Inspection of an integer-based exponent system of the forces/states, $\left[\left(v_{n^{0}} s\right)\right]^{n} \mathrm{~Hz}$ is a linear plot in the exponential domain Figures 1-4. The only other possible point values are the harmonic fractions, $\pm 1 / n_{\text {ife }}$ and harmonic mixed partial fractions, $1 \pm 1 / n_{\text {ife. }}$. These integer based exponents are associated with the observable fundamental constants. For this paper the only $n_{\text {ife }}$ utilized are points in $N_{12}$, since they are associated with the first generation of kinetic energies, particles, and bosons of neutron beta decay, Table 2, Table 3.

Assignment of the $n_{\text {ife }}$ is dependent on that value fulfilling a power law relationship with the natural unit values of the first two natural integer sets. For the simplest situation the fundamental constant is related to the $n_{\text {ife }}$ closest to 1 divided by 1 minus the exponent of the constant. If that value is positive then the partial harmonic fraction is $1-1 / n_{\text {ife. }}$. If the value is negative then the partial harmonic fraction is $1+1 / n_{\text {ife }}$. This is not the greatest integer function. However, the $n_{\text {ife }}$ value can be driven far from the closest $n_{\text {ife }}$ value by the power law imperative or the fact that some of the constants are divided or multiple by 2 . Utilizing these relationships it is possible to logically assign the physical phenomena of the neutron beta decay process to a specific partial harmonic fraction, Table 2 [7]-[14].

The simplest sinusoidal system that is related to a consecutive integer series is the possible wavelengths and frequencies of a vibrating string. This is associated with the harmonic sequence, $\{1 / n\}$, and the origin of "harmonic" in the model's name. Figure 1. Many of the properties of the model are analogous to or identical with the mathematics and physics of music. Pure number properties are important in music, and the HNH physical model such as positive or negative, even or odd, and prime, and composite. These properties also represent im- 
peratives that define the $n_{\text {ife }}$ of associated entities and the hierarchy of the physical constants.

Thus, the HNH model hypothesizes that physical constants represent a multi-layered simultaneously linear and exponential inter-locking, transformational integer series, with classic harmonic properties, in both the linear and exponential domains. These types of harmonic/repeating pattern systems are remarkably unified, where if any frequency and its associated integer value are known, then an infinite number of associated possible harmonic fractions and frequencies of the system are defined in Tables 2-4, Figure 1 of reference [14].

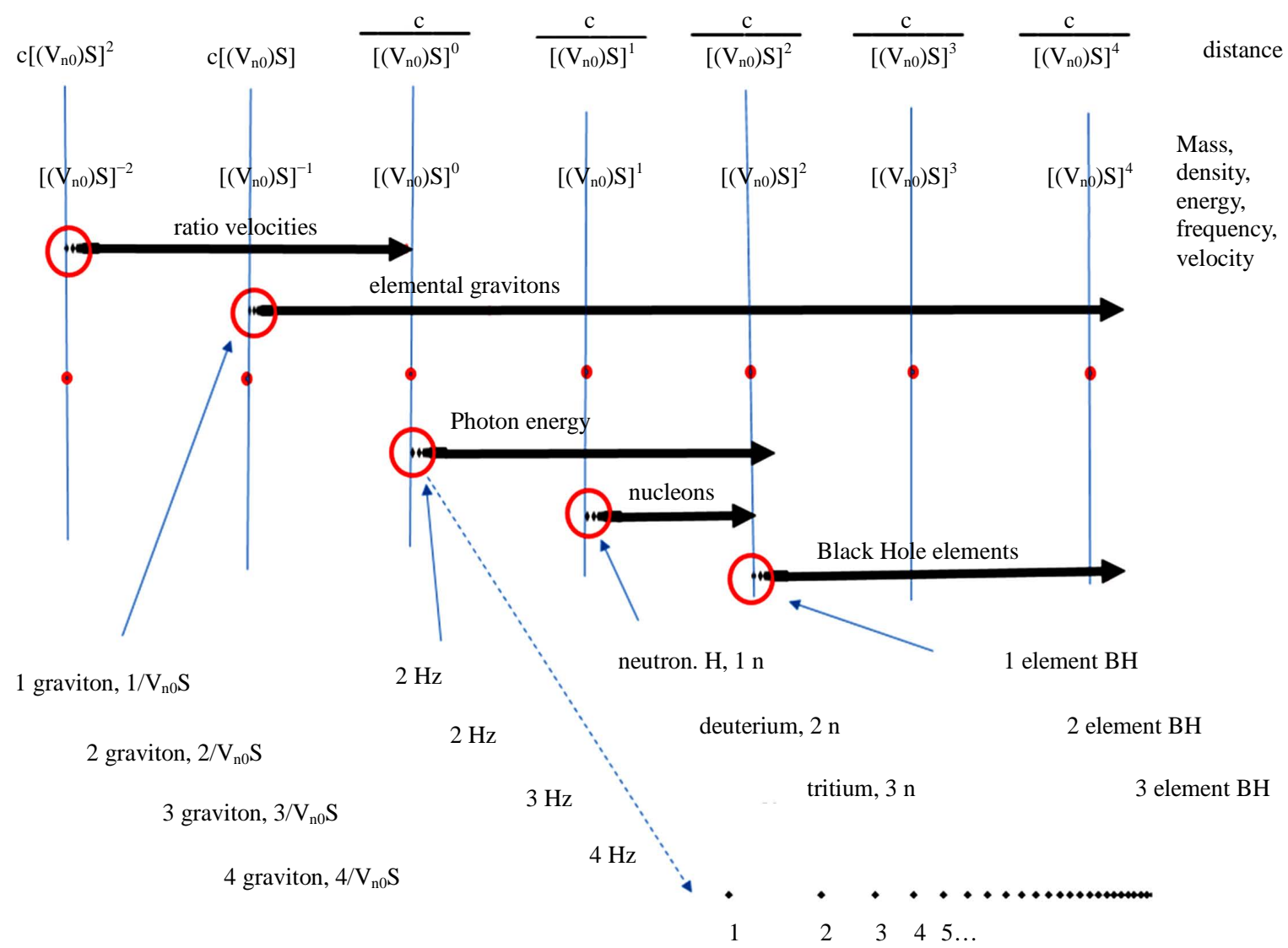

Figure 2. Combined integer point and exponential plot of the fundamental unit forces separated by a ratio of $\left(v_{n^{5}} s\right)$; multiphysical units of the $\mathrm{X}$-axis. Figure 2 is an exponential plot of the multiple $\mathrm{X}$-axis unit values and their associated degenerate integer possibilities for energy, mass, frequency, distance dimension, velocity, density, gravitational, EM, elemental strong, and black hole elements. Each is a series of $\log _{\mathrm{e}}(n)$ points starting at $n=1$ and $n$ increasing from left to right. They start as individual points, red circles, but become pseudo-continuous to the right. Each force unit value is separated by a ratio of $\left(v_{n^{0}} s\right)$, red dots and vertical blue lines. The upper row represents the distances, EM wavelengths, and mass Compton radii. Points to left of $\left[\left(v_{n^{0}} s\right)\right]^{0}$ represent possible degenerate velocity ratios $v^{2} / c^{2}$ values, of $n /\left[\left(\begin{array}{ll}v_{n^{0}} s\end{array}\right)\right]^{2}$. The points to the right of $\left[\left(v_{n^{0}} s\right)\right]^{-1}$ represent all of the possible kinetic elemental graviton energy levels, two times the gravitational binding energy of the electron in hydrogen. Points to the right of $\left[\left(v_{n^{0}} s\right)\right]^{0}$ are identical to Planck's equation E equals $n \mathrm{~Hz}$ times h, and black body radiators. The points to the right of $\left[\left(v_{n^{0}} s\right)\right]^{1}$ represent the chemical periodic chart and continues to any combination of elemental masses. The points to the right of $\left[\left(v_{n^{0}} s\right)\right]^{2}$ must represent highly dense element units of matter of black holes, $\mathrm{BH}$. 


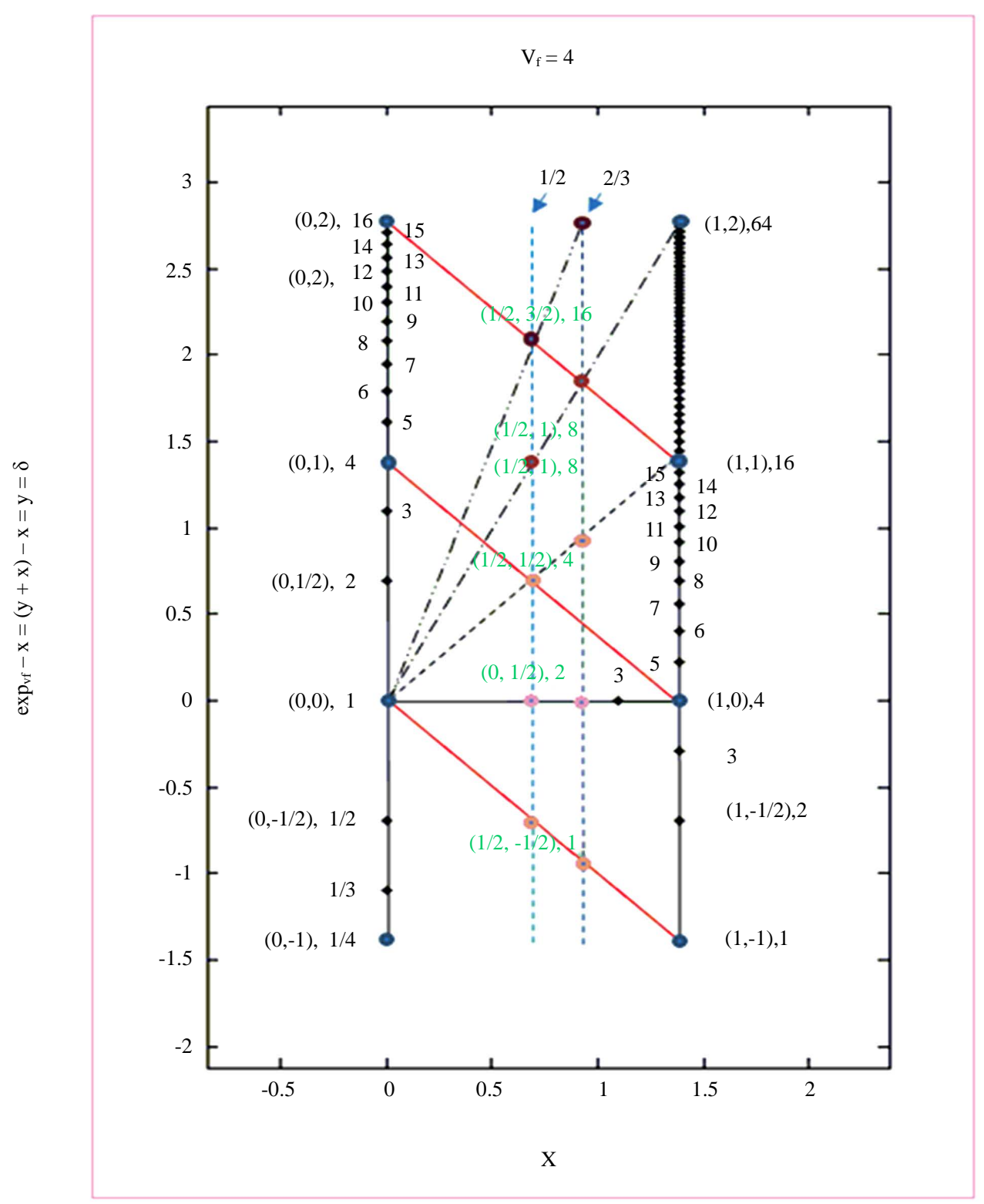

Figure 3. A universal harmonic 2d plane with an exponential base of 4 demonstrating the properties of a universal ratio, exponent, $1 / n$ calculator. Figure 3 is a $2 d$ plot of a system identical in character to the universal harmonic exponent plane, Figure 4. This is a simplified example where the $V_{f}=4$. This is both an integer and exponential series. The number, 4, is chosen to illustrate the nature of this $2 \mathrm{~d}$ space in a comprehensible fashion. This $2 \mathrm{~d}$ space represents a very powerful geometry that is similar to a slide rule, but with infinite flexibility. This includes all of the possibilities that cannot be displayed on a line plot. The blue points represent the only valid integer exponents of $V_{f}$. In this case any absolute sum of an $x$ and $y$ vector that adds to 1 is a potentially valid integer exponent point. The only other possible valid points are those that fall on lines that connect between two integer points, blue dots. Any absolute $x$ plus $y$ orthogonal distance of 1 is identical to multiplying or dividing that number by 4 in the frequency domain. Therefore an absolute unit distance from the $(0,0)$ point forms a diamond pattern. This is identical to the actual physical constants seen in Figure 4 from [7]. The exponent of $V_{f}$ equals $x+y$ at any point. The red lines are identity lines, all with the same exponent and frequency. The $\delta$-values represent the exponent minus the $x$ value, or $(x+y)-x$, and therefore equals $y$. The black solid and dashed lines all converge on $(0,0)$, which equals a frequency of 1 . In this specialized case these lines define $v_{f}$ of $1,4,16$, and 64 . The vertical dashed blue lines represent the fractional exponents of each $v_{f}$ of $1 / 2$ and $2 / 3$. The X-axis literally represents any exponent. In a continuous system any exponent of any $V_{f}$ represents the intersection of a vertical line at $x$, and the line connecting $(0,0)$ to any $V_{f}$ value at $x$ equals 1 . The values of any point on a line in this space is defined totally from the perspective of $V_{f}$. Two points can define any ratio, and simultaneously define any $\delta$-line. Lines not passing through $(0,0)$ also define a well-defined pattern, but are more complicated to describe. 


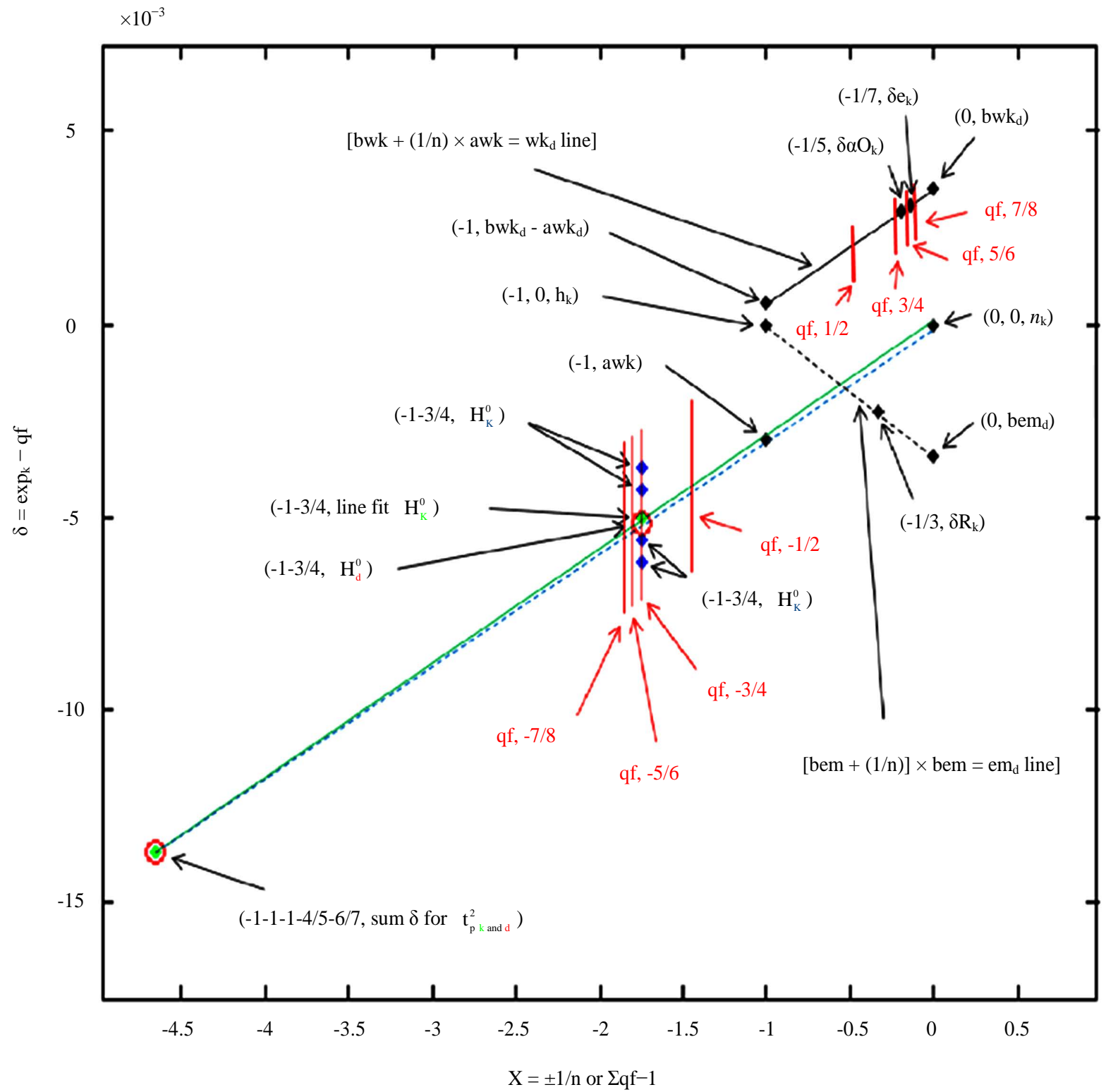

Figure 4. Universal 2d harmonic plane plot of the known and derived subatomic entities, $t_{p}^{2}, H_{0}$. Figure 4 is a universal harmonic exponent plot of the relevant physical constants. The X-axis equals the $q f-1$ or sum of $q f_{d}-1$. The $Y$-axis is the difference between the known or derived exponents and their $q f s$, and $\delta s$ known or derived. This is a power law test of the model. The slopes and y-intercepts of the three lines are sums and differences of the three published values. The previously published points related to $h, n^{0}, e, R, \alpha_{0}, t_{p}^{2}$ are plotted. These points define three lines. The electron and $\alpha_{0}$ define the wk-line with slope of awk and $y$-intercept, bwk (blue solid line). The EM line is defined by $h$ and $R$ (dashed blue line). The green dashed line is the derived $\delta_{d}$ line for $t_{p}^{2}$. The known $t_{p}^{2}$ point is plotted as a green dot. The derived $t_{p}^{2}$ point is centered at the red circle and the derived line fit value are all superimposed. The blue points are four of the experimental values for $H_{0}$ with their associated $\delta_{k}$. These are plotted at the $x$-value of $-3 / 4-1$ since the derived $q f=-3 / 4$. The line fit of the experimental $t_{p}^{2}$ point and the four $H_{0}$ points is plotted at the green dot. The solid green $\delta_{k}$-line is the best fit of the experimental data and closely parallels the derived $\delta_{d}$-line. The experimental solid green $\delta_{k}$-line fit data for $t_{p}^{2}$ and $H_{0 k}$ are very close to their derived values, the dashed green line, Table 1 . None of the other $q f s$ of $-1 / 2,-5 / 6$, or $-7 / 8$ are within orders of magnitude of the $H_{0}$, and do not represent valid $q f s$ for $H_{0}$. The vertical thin red lines are associated with the hypothesized possible negative even harmonic fractions, $-1 / 2,-3 / 4,-5 / 6,-7 / 8$. The thick vertical red lines are the values along the wk-line used to derive the energy lost in the neutron beta decay process, af $+1 / 2,+3 / 4,+5 / 6$, and $+7 / 8$. 
Table 3. List of natural units used in the derivations. Table 3 lists the published values of $\left(v_{n^{5}} s\right)$, and the slopes and intercepts of the wk and EM lines used for the derivation of $H_{0}$. These are the only natural values used for all of the derivations. See Equations (13)-(15).

\begin{tabular}{lc}
\hline \multicolumn{1}{c}{ Physical constant } & Value \\
\hline$\left(v_{n^{0}} s\right)$ & $2.271859078(50) \times 10^{23}$ \\
$\log _{e}\left[\left(v_{n^{0}} s\right)\right]$ & $53.780055612(22)$ \\
bwk: $y$-intercept, weak force, wk line & $3.51638329(18) \times 10^{-3}$ \\
awk: slope, weak force, wk line & $3.00036428(15) \times 10^{-3}$ \\
bem: $y$-intercept, electromagnetic, EM line & $-3.45168347(17) \times 10^{-3}$ \\
aem: slope, electromagnetic, EM line & $-3.45168347(17) \times 10^{-3}$ \\
\hline
\end{tabular}

Table 4. Comparison of derived and known proportionality constants of $H_{0}, t_{p}^{2}$, and $n^{0}$. Table 4 is a comparison of derived and now proportionality constants of $H_{0}, t_{p}^{2}$, and $n^{0}$. The derived values fall within the range of the known values.

\begin{tabular}{ccc}
\hline Proportionality constant & Known & Derived \\
\hline$H_{0 d} /\left[\left(v_{n^{0}} s\right)\right]$ & $1.02(15) \times 10^{-41}$ & $1.01118370(17) \times 10^{-41}$ \\
$t_{P}^{2} /\left[\left(v_{n^{0}} s\right)\right]$ & $8.03(40) \times 10^{-110}$ & $8.0382241(5) \times 10^{-110}$ \\
$H_{\text {od }} / t_{P}^{2}$ & $1.272(64) \times 10^{68}$ & $1.25796898(7) \times 10^{68}$ \\
\hline
\end{tabular}

\subsection{Previous Derivations of the $\mathrm{HNH}$ and the Relationship to the Derivation of the Hubble Constant}

The Harmonic Neutron Hypothesis was previously used to derive the energy/matter lost in the transformation of a neutron to hydrogen, the masses of the quarks, the Higgs boson, and the Planck time [9] [10] [14]. Purely kinetic factors of neutron beta are related to the even-numbered denominators in the harmonic fractions, $1 / 2,3 / 4$, $5 / 6$, and $7 / 8$. It is logically hypothesized that the additive inverse partial harmonic fractions, which includes $-1 / 2$, $-3 / 4,-5 / 6$, and $-7 / 8$ should be related to the cosmic kinetic fundamental constants including, the $H_{0}$, cosmic microwave background radiation, $\mathrm{CMB}$, and dark matter/dark energy.

The HNH has accurately derived Planck time, $t_{p}$, from this same sets of integers. [9]. It is also logical to hypothesize that other cosmic fundamental constants should be related to $t_{P}$. Our derivation shows that $t_{P}^{2} \times s^{2}$ is identical to the Newtonian gravitational constant, $G$, in the frequency domain. The product of $t_{P}^{2} \times s^{2}$ and the frequency equivalents of two masses and distance separating them equals the gravitational binding energy as a frequency equivalent of that system. The derived values associated with the $t_{P}^{2}$-line at the partial harmonic fractions $-1 / 2,-3 / 4,-5 / 6$, and $-7 / 8$ should be related to the cosmic kinetic fundamental constants including $H_{0}$, cosmic microwave background radiation, CMB, dark matter and energy.

\subsection{Similarities of the HNH and Magnetic Resonance Imaging}

None of the individual elements of the HNH are new or radically depart from standard physics or mathematical methods. The perspective taken, the nomenclature used, and methods are not standard, but are nonetheless logically and mathematically valid. To understand this method, a significant intellectual investment is essential since it is not intuitively obvious. The model is from a global perspective very similar to magnetic resonance imaging. MRI, which was assumed to be impossible at the time of its introduction based on classical physics interpretations of optical imaging criteria, yet disproven [15] [16]. Both MRI and the HNH are spectral analysis methods. Both have two different domains that simultaneously define the identical object/system. One domain is in standard linear physical 3d space, and the other is in a mathematically transformed domain related to phase and fre- 
quency, the k-space for MRI. In this model it relates to an exponential universal harmonic plane, Figures 2-4. Both represent the identical physical system, but are defined in different mathematical terms.

\subsection{Why the Harmonic Neutron Hypothesis Is Based on Classical Physics and Not Numerology}

The standard components of the HNH will be highlighted in italics. The HNH model is not in conflict with the Standard Model or overturns its methods or tenants. The actual physical values used are equivalent to standard unit values, but they are all transformed into frequency; harmonic fraction plus $\delta$; or exponential equivalents. They are evaluated as dimensionless coupling constant ratios, and proportionality constants. For example the ratio of the mass of the electron divided by the mass of the neutron equals the frequency equivalent of the electron divided by the frequency equivalent of the neutron independent of unit.

This model is independent of any specific physical unit system. Converting the standard units to this unit 1 format does not change the absolute $V_{f}$ integer value, The reason for this non-intuitive mathematical imperative is that any arbitrary mass unit changes the arbitrary Avogadro's number, $N_{A}$, canceling out any effect of the initial mass unit. Transforming the arbitrary distance, time, and speed of light units to 1 are canceled out by changing the unit distance ratio with the fixed Compton radius, and changing the time unit to 1 . Time and distance in the speed of light are proportional so the changes parallel each other, and cancel out any choice of those initial arbitrary values.

The combined components of the hypothesis are controversial because they are not well understood, are different from standard nomenclature, and novel. The concepts and mathematics are actually not complicated, but require a significantly different conceptual approach. All of the physical relationships are viewed solely as exponents (integer fractions plus $\delta$ ) or ratios of $\left[\left(v_{n^{0}}\right) s\right]$ In the linear domain these are ratios, coupling constants/proportionality constants. One example would be the ratio of the frequency equivalent of the ionization energy of hydrogen divided by the frequency equivalent of the Bohr Radius, $\alpha_{0}$ [11]. This is equivalent to Coulomb's constant in the frequency domain.

All of the ratios are scaled by $\left(v_{n^{0}}\right) s, V_{f}$, and therefore proportionality constants. Therefore, in this paper there should be a derivable proportionality constant that inter-relates $t_{P}^{2}, n^{0}$, and $H_{0}$, Table 4. Presently there is no known proportionality constant relating these three physical constants.

In this model the identical consecutive integer and harmonic fraction sequences are seen, as exponents of the fundamental frequency, $V_{f}$, of the system, Figures 1-3. The origin of this exponential pattern is not from a standing wave sinusoidal pattern, but from a consecutive integer exponential sequence defining the forces. In this type of ratio system, when $V_{f}$ is raised to an exponent in the sequence, the results represent the only possible observable frequencies of the derived or known physical constants. When this sequence of points is plotted on a 2d-plane, the only possible exponents are the integer, the harmonic fraction, and the partial fractional sequence of $1 / n$ and $1 \pm 1 / n$, where $n$ equals $N_{12}$, Figure 3, Figure 4. The resulting 2d-plane represents a universal 2d-exponential "slide rule", Figure 3 [17].

The hypothesis is based on classic harmonic fractions, $\{ \pm 1 / n\}$ and partial harmonic fractions, $1 \pm 1 / n$ for $n=\{1,2,3, \cdots\}$. This offers a tremendously powerful, predictive attribute of the model since associating a physical constant with a specific harmonic fraction creates a series of other discrete harmonics and characterizes the whole system from an initial two-element data set. This is how $H_{0}$ can be derived with no direct physical measurement. An analogy is Moseley's law on ion emission spectra.

Another important concept is resonance and products of harmonic numbers. Resonance is the tendency of a system to oscillate with greater amplitude at some frequencies rather than at others. If two systems have common frequency components there will be greater coupling and potential transfer of energy between them. For a musical example, two prime number frequencies can only resonant at the product of the two prime frequencies. This pure number property defines a higher order hierarchy of physically associated entities.

The HNH presents itself as a natural unit system. A natural unit system incorporates known physical units rather than arbitrary units. A natural unit model with all of the other constants driven to 1 greatly simplify the mathematics. This model is based on the annihilation frequency of the neutron, as the fundamental frequency similar to Planck units. In Planck units all of the different fundamental constants are converted into a single common standard unit such as $\mathrm{Hz}$, seconds, kilograms, or meters. The neutron is a logical unified fundamental 
physical entity that is centered between atomic, subatomic, and cosmic entities.

\subsection{Why $\left[\left(v_{n^{0}}\right) s\right]^{(1 \pm 1 / n)}$ Are Not the Exact Fundamental Constants' Physical Values, and Why Are $\delta$-Values Essential?}

In the simplest exponential harmonic series, all of the possible frequency values could be defined and related solely to a single fundamental frequency and the harmonic fraction series, Figure 2, Figure 3 [14]. The HNH and physical reality are far more complicated. This arises from a mathematical imperative. Known fixed number values of the products of 2 and pi are associated with specific exponent integer-fraction values. These arise from the product ratio relationships of $R, \alpha_{0}, e$, and, $\alpha$ [11].

There are four product ratio relationships of these entities associated with the hydrogen atom. For example the integer fraction associated with 2 must be related to $\left[\left(v_{n^{0}}\right) s\right]$ raised to (10/1155). Also 2pi must be related to $\left[\left(v_{n^{0}}\right) s\right]$ raised to (39/1155). Additionally, 2 raised to (1/(10/1155)) is $5.8744 \times 10^{34}$, and (2pi) raised to $(1 /(39 / 1155))$ is $4.34916 \times 10^{23}$. There is no common fundamental frequency that can fulfill these conflicting mathematical imperatives. Nature's solution is to have small $\delta$-values added to the quantum harmonic fractions that "shim" these various values to a common fundamental frequency, in this case $\left(v_{n^{0}}\right) \mathrm{Hz}$. These $\delta$-values represent the known or derived exponent minus the harmonic fraction. This makes the system more complicated, but fulfills the imperative of a resonant system.

\subsection{Computations Using the Universal Harmonic 2d Exponential Plane}

Each physical constant is plotted as a harmonic fraction minus one on the $\mathrm{X}$-axis and the $\mathrm{Y}$-axis is the plot of $\delta$ (the exponent minus the harmonic fraction), Figure 3, Figure 4. This is described as the universal harmonic plane. It is universal since it can perform any ratio, product, or power calculation, and all of the physical constants are plotted on this common 2d space. [7] [14] [17]. It is harmonic since the X-axis is defined by sums and differences of integers and harmonic fractions only.

The physical datum of each point has the identical value as its standard exponent, and can be translated to its standard routine physical value. The difference between two points on the $2 \mathrm{~d}$ universal plane represents a proportionally constant, a ratio in the linear domain, a power law. This harmonic plane also has all of the classic mathematical properties of lines. A line connecting any two points can define a proportionality relationship of two or more physical constants. It is possible to derive any harmonic value from the slope and y-intercept of a $\delta$-line and $\left(v_{n^{0}}\right)$, if the harmonic fraction is known or derived. Different forces can be associated with different or common $\delta$-lines.

\subsection{Derivation of Experimentally Unmeasured Physical Constants}

In Physics, under most circumstances if one knows a natural unit within a ratio or product relationship that value can be used as a constant, but it does not have inherent predictive value to other multiple other related physical constants. In a harmonic system if the natural unit is known and its associated quantum integer value then an infinite series of other values/constants, can potentially be derived. [14] This is a classic property or quantum spectrum.

Many of these constants can be derived since the actual $\delta$-values can be derived from the three finite point sets described above sets, provided the harmonic fraction is logically derived. [7]. The data define two lines on the universal harmonic plane. Their slopes and y-intercepts along with $\left(v_{n^{0}}\right) s$ are all that is necessary to derive many of the other physical constants in this model. One line is related to the weak kinetic entities, and one related to the electromagnetic entities. The only other possible valid force $\delta$ lines are related to sums and differences of the slopes and y-intercepts of those two lines, Figure 4. Harmonic fractions, other than the very limited set used here, opens the possibility to derive the actual $\delta$-values since they would represent just another point on a valid line following the power law and quantum spectrum properties. 


\subsection{Value of the HNH Method and New Insights into the Meaning and Origin of Hubble's Constant}

The value of the HNH method is that it can derive and predict physical constants beyond what can be experimentally measured [9] [10] [14]. The Standard Model today cannot unify or scale quantum and cosmic phenomena simultaneously. The HNH also demonstrates the inter-relationship of the constants. The derived values have high precision since the calculations are based on high precision atomic data to begin with, and not on experimental data related to the physical constant in question [14]. In the present paper no astronomical data is utilized in the derivation of $H_{0}$.

The methodology of $\mathrm{HNH}$ derivation generates new insights into connections between the subatomic entities of neutron beta decay, kinetic energy, and the neutrinos, leading to frequency expressions of the neutron, $t_{P}$, gravity, $H_{0}$; and the apparent expansion of the universe.

\section{Methods and Results}

\subsection{Conversion of Physical Constants to Frequency Equivalents}

Floating point accuracy is based upon known experimental atomic data, of approximately $5 \times 10^{-8}$. All of the known fundamental constants are converted to frequency equivalents, $v_{k},\{k=1, \cdots, 4\}$, for the four (4) physical constants used in the HNH derivation of $H_{0}$. Equations (1)-(4), Table 3. The masses are converted by multiplying by $c^{2}$ (speed of light squared) then dividing by h (Planck's constant). The distances are converted by dividing the wavelength into $c$. Energies in Joules are converted by dividing by $\mathrm{h}$. The $\mathrm{eV}$ value for the neutron is $939.565378(21) \times 10^{6}$. Its frequency in $\mathrm{Hz}$ is converted to $\mathrm{eV}$ by multiplying by the constant, $4.13566750(21) \times$ $10^{-15} \mathrm{eV} / \mathrm{Hz}$. The $\mathrm{eV}$ was converted to frequency by multiplying by the constant $2.41798930(13) \times 10^{14} \mathrm{~Hz} / \mathrm{eV}$. $N_{A}$ is Avogadro's number, 6.02214129(27) $\times 10^{23} \mathrm{~mol}^{-1}$. Converting the standard units to where they are all 1's does not change $V_{f}$.

$$
\begin{aligned}
& v_{n^{0}}=\frac{c^{2}}{h} m_{n^{0}}=2.271859078(50) \times 10^{23} \mathrm{~Hz} \\
& v_{n^{0}}=\left(\frac{c^{2}}{h}\right)\left(\frac{10^{-3}}{N_{A}}\right)\left(1.00866491600 \frac{a m u}{n^{0}}\right)=2.271859078(50) \times 10^{23} \mathrm{~Hz} \\
& v_{e}=\frac{m_{e} c^{2}}{h}=1.2355899(1) \times 10^{20} \mathrm{~Hz} \\
& v_{\alpha_{0}}=\frac{c}{\alpha_{0}}=5.6652564(3) \times 10^{18} \mathrm{~Hz} \\
& v_{R}=c R=\frac{c}{1 / R}=3.2898419(17) \times 10^{15} \mathrm{~Hz}
\end{aligned}
$$

All of the data for the fundamental constants were obtained from the websites (http://physics.nist.gov/cuu/Constants/ and www.wikipedia.org. The NIST site http://physics.nist.gov/cuu/Constants/energy.html has an online physical unit converter that can be used for these types of conversions.

\subsection{The Frequency Equivalent and Exponential Domains; Calculation of Known Exponents, $\exp _{k}, n_{\text {ifek, }} q f_{k}$, and Known $\delta_{k}$ Values from Frequency Equivalents}

This model has two parallel domains both describing the identical physical values. One domain is the frequency equivalent of any physical value. The other domain is the exponent of the base $\left[\left(v_{n^{0}}\right) s\right]$, which when raised to that exponent equals the frequency equivalent of that specific value. Equation (5). The known exponent, $\exp _{k}$, of a fundamental constant is the ratio of the $\log _{e}$ of the frequency equivalent, $n_{k}$ s, divided by the $\log _{\mathrm{e}}\left[\left(v_{n^{0}}\right) s\right]$, 
Equation (5). Here, $\log _{\mathrm{e}}\left[\left(v_{n^{0}}\right) s\right]=53.780055612(22)$. Subscript $k$ denotes a known experimental value and subscript $\mathrm{d}$ represents a derived value.

$$
\exp _{k}=\frac{\log _{\mathrm{e}}\left(v_{k}\right)}{\log _{\mathrm{e}}\left(v_{n^{0}} s\right)}=\log _{v_{n^{0}} s}\left(v_{k}\right)=q f_{k}+\delta_{k}
$$

Every value in the physical domain is defined completely in terms of its ratio and exponent relationship with $\left[\left(v_{n^{0}}\right) s\right]$. All other constants are 1. Each domain describes the identical physical value, but one does so in the standard physics linear/frequency domain, and the other in the exponential domain which is unique to the HNH model. The value of viewing the fundamental constants in the exponential domain is that their harmonic integer inter-relationships are clearly defined. The other is that any physical relationship can be displayed and calculated across all of the forces, and at any scale in this virtual 2d space. Despite the fact the HNH model utilizes virtual space, that space accurately defines true physical phenomena.

In the Standard Model, only a subset of physical values are quantum by definition or computational in character. In the HNH model every aspect of physical systems is quantized by integral steps. There are regions where the system appears to be experimentally continuous in the Standard Model, but this is not true in the HNH model, Figure 1. In the Standard Model there are physical values that equal 0, such as a velocity, but in the $\mathrm{HNH}$ this is not the case. In regions that are A.E. continuous the true quantum values can be evaluated in the HNH model.

The known $\exp _{k}$ minus the harmonic/quantum fraction, $q f$, equals the known $\delta_{k}$, Equation (6). The known frequency equivalent of a constant, $v_{k}$, is calculated by raising $\left[\left(v_{n^{0}}\right) s\right]$ to the $\exp _{k}$ in Equations (6). Equation (7) shows that many of the fundamental constants do not have $n_{f}$ equal to $\left(v_{n^{0}}\right) s$, but $\left[\left(v_{n^{0}}\right) s\right]^{(1+\delta @ x=0)}$.

$$
\begin{aligned}
& y_{k}=\delta_{k}=\exp _{k}-q f_{k} \quad \text { or } \quad \exp _{k}=q f_{k}+y_{k}=q f_{k}+\delta_{k} \\
& v_{k}=\left(v_{n^{0}} s\right)^{\exp _{k}}=\left(e^{53.7800561(32)}\right)^{\exp _{k}}=\left(v_{n^{0}} s\right)^{q f+\delta_{k}}=\left(v_{n^{0}} s\left(1+\frac{\delta_{k}}{q f}\right)\right)^{q f}=\left(v_{n^{0}} s^{(1+\delta @ x=0)}\right)^{q f}
\end{aligned}
$$

The $n_{\text {ife }}$ (integer-fractional exponents, "ife") and the associated quantum fractions, $q f$, the harmonic fractions, and the partial harmonic fractions must fulfill a power law relationship with a natural unit constant. The closest $n_{\text {ife }}$ to the experimental value is derived from the $\exp _{k}$, Equations (8), but that value may not be the actual $n_{\text {ife }}$ since it may not fulfill a power law relationship [10]. The first $n_{\text {ife }}$ assignments from 1 to 12 are listed in Table 2 . The higher principal quantum number values are harder to assign unless the constant is known with a high precision. There have been errors in assignment in the past, for example, the strange quark was initially assigned to the qf 29/30, but later corrected to 27/28, [10] [14].

$$
\begin{aligned}
& n_{\text {ife }}=\text { closest } n_{\text {ife }} \text { to } \frac{1}{1-\exp _{k}} \\
& \text { if } \frac{1}{1-\exp _{k}}>0 \text { then } \text { qf }=1-\frac{1}{n_{\text {ife }}} \text {, if } \frac{1}{1-\exp _{k}}<0 \text { then } \text { qf }=1+\frac{1}{n_{\text {ife }}}
\end{aligned}
$$

\subsection{Association of Individual Physical Constants to Harmonic Fractions and Their Degenerate Frequency Equivalents}

As $\left[\left(v_{n^{0}}\right) s\right]$ is raised to exponents of a consecutive harmonic quantum fraction (qf) $\{1 \pm 1 / n\}$ for $n=\{1,2,3, \cdots\}$ it represents many of the degenerate exponent values of the fundamental constants, Equations (9)-(11). The degenerate ratios of the constant's frequency when divided by $\left(v_{n^{0}}\right) s$ represent $\left(v_{n^{0}}\right) s$ raised to 
the quantum fractions $q f$, where the harmonic quantum fraction exponents equal $1 / \pm n$ where the natural number, $n=\{1,2,3\}$. These represent the degenerate proportionality constants relating $\left(v_{n^{0}}\right) s$ to the degenerate $n$ value.

$$
\begin{aligned}
& \text { qf }=1 \pm \frac{1}{n_{\text {ife }}}=x+1 \text { for principal quantum number } n_{\text {ife }}=1 \text { to } \infty \\
& v_{\text {degenerate }}=\left(v_{n^{0}} s\right)_{\text {degenerate }}^{(q f)}=\left(v_{n^{0}} s\right)_{\text {degenerate }}^{1 \pm \frac{1}{n_{\text {fife }}}} \text { for principal quantum number } \\
& \frac{v_{\text {degenerate }}}{v_{n^{0}} s}=\left(v_{n^{0}} s\right)_{\text {degenerate }}^{\left(\frac{1}{n_{\text {ife }}}\right)} \text { for principal quantum number }
\end{aligned}
$$

Equation (12) calculates the $\mathrm{X}$-axis value for a specific quantum fraction, partial fraction, and $n_{\text {ife. }}$.

$$
x=q f-1=\left(1 \pm \frac{1}{n_{\mathrm{ife}}}\right)-1= \pm \frac{1}{n_{\mathrm{ife}}}
$$

\subsection{Calculation of Known Exponents, $\exp _{k}$ and $\delta_{k}$ Values for $n^{0}, e, \alpha_{0}, R, \alpha^{-1}, h$}

By definition the $\exp _{k}$ of the $n^{0}$ is 1 , and the $\exp _{k}$ of $\mathrm{h}$ is 0 . Both have a $\delta_{k}$ of 0 . All of the electromagnetic spectrum, quantized $n-\mathrm{Hz}$, have an effective $\delta=0$ (i.e. degenerate). All photons are actually not degenerate, but split with a Lorentz factor of $V_{f}$ for energy and Lorentz factor of $1 / V_{f}$ for time dilation. The other quantized numbers used for $e, \alpha_{0}, R, \alpha^{-1}$ are related to the first four odd prime numbers. The frequency equivalent of the $\alpha_{0}, \quad v_{\alpha_{0}}$, is $5.66525639(28) \times 10^{18} \mathrm{~Hz}$; where $\exp _{\alpha_{0}}$ is $0.80291631(05)$. The $q f$ is $4 / 5$, on the $\mathrm{X}$-axis location of $-1 / 5$, with principal quantum number 5 , and its $\delta_{\alpha_{0}}$ equals $2.91631043(14) \times 10^{-3}$. The exponent $V_{f}$ of $\alpha_{0}$ is $1+3.6453880(02) \times 10^{-3}, b_{\alpha_{0}} 3.6453880(02) \times 10^{-3}$, and $v_{f}$ of $2.76391359(14) \times 10^{23} \mathrm{~Hz}$. The $y$-intercept of $v_{f}$ line is referred to as the $b$ entity $\delta$ value, in this case $b_{\alpha_{0}}$.

The frequency equivalent of the electron $n_{e}$, is $1.23558996(05) \times 10^{20} \mathrm{~Hz}$; $\exp _{e}$ is 8.6023061(06). The $q f$ is 6/7, $\mathrm{X}$-axis location of $-1 / 7$, principal quantum number, 7 , and its $\delta_{e}$ is $3.08775982(21) \times 10^{-3}$. The exponent $v_{f}$ of e is $1+3.60238646(18) \times 10^{-3}$, be 3.60238646(18) $\times 10^{-3}, v_{f}$ of $2.7575290(01) \times 10^{23} \mathrm{~Hz}$.

The frequency equivalent of hydrogen ionization energy $v_{R}$, is $3.28984196(17) \times 10^{15} \mathrm{~Hz}$; $\exp _{R}$ is 6.64365544(33). The of is $2 / 3$, X-axis location of $-1 / 3$, principal quantum number 3 , and its $\delta$ is $-2.30112231(11) \times 10^{-3}$. The exponent $v_{f}$ of $R$ is $1-3.45168347(17) \times 10^{-3}, b_{R}-3.45168347(17) \times 10^{-3}, v_{f}$ of $1.88695938(09) \times 10^{23} \mathrm{~Hz}$.

The frequency equivalent of $\alpha^{-1}$ is 1.370359991(69) $\times 10^{2}$; $\exp _{\alpha-1}$ is $9.14882590(46) \times 10^{-2}$. The qf is $1 / 11$, X-axis location of $-10 / 11$, principal quantum number 11 , and its $\delta_{k}$ is $5.7916811(03) \times 10^{-4}$.

\subsection{Plotting, Transformation, of Known Exponents and $\delta_{k}$ Values on to the $2 \mathrm{~d}$ Universal Harmonic Exponential Plane for $\boldsymbol{n}^{0}, \boldsymbol{h}, \mathrm{e}, \alpha_{0}$, and $\boldsymbol{R}$}

The known and derived exponents of physical constants are plotted/ transformed to the $2 \mathrm{~d}$ universal harmonic plane. The $\mathrm{X}$-axis is a multi-dimensional physical descriptor, Figures $2-4$. The point $(0,0)$ represents the neutron since that is the exponent of $v_{n^{0}} \mathrm{~Hz}$ divided by $v_{n^{0}} \mathrm{~Hz}$. The $\mathrm{Y}$-axis is the related to the difference of the known exponent minus its associated $q f$, Equation (6). The Y-axis is not continuous in terms of the inter-relationships of the fundamental constants either. The slopes and y intercepts of the wk and EM $\delta$ lines also represent three "quantum" unit values though not integers. The only possible $\delta$-values are a function of qf- 1 , and are discrete.

The points for the $e, \alpha_{0}$ and $\alpha^{-1}$ fall above the $\mathrm{X}$-axis since their $\delta_{k}$ are positive. Their respective $\mathrm{X}$-axis values are $-1 / 7=(6 / 7-1),-1 / 5=(4 / 5-1),-10 / 11=(1 / 11-1)$. The point for $R$ falls below that $\mathrm{X}$-axis at $x$ equals $-1 / 3=(2 / 3-1)$ since the $\delta_{k}$ is negative. The differences between these points on the universal harmonic exponent plane represent the classic ratios of these same values in the frequency domain [12]. 


\subsection{Calculation of Derived Exponents, $\exp _{d}$, Derived $\delta_{d}$ Values; and Calculation of Derived $\delta_{d}$ Slopes and Intercepts at $x=-1$ and $x=0$}

The only possible derived exponents are discrete since the only possible $\mathrm{qf}$ and $\delta$ values are discrete. The lines described below transpose the natural unit sets to the harmonic $2 \mathrm{~d}$ plane. The line connecting the $e,\left(-1 / 7, \delta_{e}\right)$, and $\alpha_{0},\left(-1 / 5, \delta_{\alpha_{0}}\right)$, points is logically related to the weak kinetic force, $w \mathrm{k} \delta_{d}$-line. Here, $\alpha_{0}$ is a distance and related to a length dimension and velocity, therefore kinetic properties. The electron is a mass related to the weak force. The other $1 / \pm n$ points on the wk line represent the other possible derived $\delta_{d} \mathrm{~s}$ that are logically related to the weak force. These possibilities graphically represent the intersection points of the $w \mathrm{k} \delta_{d}$ line with $x$ values of $\pm 1 / n$. The slope is awk, 3.000364286(15) $\times 10^{-3}$ and the $y$-intercept is bwk, 3.51638329(18) $\times 10^{-3}$, Equations (13), (14).

$$
\begin{aligned}
\mathrm{awk} & =\frac{\left(\exp _{e}-\frac{6}{7}\right)-\left(\exp _{\alpha_{0}}-\frac{4}{5}\right)}{\left(\frac{6}{7}-\frac{4}{5}\right)}=3.000364286(15) \times 10^{-3} \\
\mathrm{bwk} & =\left(\exp _{e}-\frac{6}{7}\right)+(a w k) \times \frac{1}{7}=\left(\exp _{\alpha_{0}}-\frac{4}{5}\right)+(a w k) \times \frac{1}{5}=3.51638329(18) \times 10^{-3}
\end{aligned}
$$

These derived $\delta_{d} w k$ values when proposed had no known physical significance. [7] It was assumed that these other "possible valid" harmonic fraction points along a $\delta_{d}$ line are identical in concept and physical reality to other possible "spectral lines" in a standard quantum spectrum where each spectral line is associated with a different quantum integer. In the interval these hypothesized $\delta_{d}$ points have been shown to be associated with many known physical entities. [9] [10] [14]

The EM, line is defined by the points for the Planck constant, $(-1,0)$ and Rydberg $R\left(-1 / 3, \delta_{R}\right)$, Figure 4 , Equation (15). This is the second line that was previously published, [7]. This is logical since $\mathrm{h}$ is the unit for electromagnetic energy, and $R$ is the unit for the atomic ionization energy. It is referred to as the electromagnetic, EM $\delta_{d}$-line. Its slope and y-intercept, bem, are identical and equal to $-3.45168347(17) \times 10^{-3}$, Equation (15). This line is related to the principal quantum number $3, q f, 2 / 3$. The other potential $q f$ values have also been shown to be related to the quarks and mesons, [10].

$$
\text { bem }=\frac{3}{2}\left(\exp _{R}-\frac{2}{3}\right)=-3.45168347(17) \times 10^{-3}
$$

The derived $\delta_{d}$ values are a linear function of the qf-1, and the slope and y intercepts of the $\delta_{d}$-lines. An alternate definitions of slope and y intercept are the $y$ values at $x$ equals 0 , and -1 , Equations (16). These are interchangeably equivalent, and both are used in this method, Figure 4. It is possible to predict the exact exponents of unmeasured fundamental constants from these $\delta_{d}$ lines just like it is possible to derive lines of quantum spectrum without a physical measure if its natural unit and principal quantum number are known, Equation (17), (18).

$$
\begin{aligned}
& y_{d}=\delta_{d}=\left(\operatorname{slope}_{\delta \text { line }}\right)\left(x_{d}\right)+y_{d \delta \text { line } @ x=0}=\left(\delta_{d @ x=0}-\delta_{d @ x=-1}\right)\left(x_{d}\right)+\delta_{d @ x=0} \\
& y_{d}=\delta_{d}=\left(\text { slope }_{\delta \text { line }}\right)\left(q f_{d}-1\right)+y_{d \delta \text { line@ } @=0}=\left(\delta_{d @ x=0}-\delta_{d @ x=-1}\right)\left(q f_{d}-1\right)+\delta_{d @ x=0} \\
& y_{d}=\delta_{d}=\left(q f_{d}\right)\left(\delta_{d @ x=0}\right)+\left(1-q f_{d}\right) \delta_{d @ x=-1} \\
& \exp _{d}=\log _{v_{n} \varsigma}\left(v_{d}\right)=q f_{d}+\delta_{d}=q f_{d}+\left(q f_{d}-1\right)\left(\delta_{d @ x=0}-\delta_{d @ x=-1}\right)+\delta_{d @ x=0} \\
& \exp _{d}=\log _{v_{n} s}\left(v_{d}\right)=q f_{d}+\left(x_{d}\right)\left(\text { slope }_{d \delta \text { line }}\right)+y_{d @ x=0}=q f_{d}+\left(q f_{d}-1\right)\left(\text { slope }_{d \delta \text { line }}\right)+\delta_{d @ x=0} \\
& v_{d}=\left(v_{n} s\right)^{\exp _{d}}=\left(v_{n} s\right)^{q f_{d}+\delta_{d}}=\left[v_{n} s^{\left(1+\delta_{d} @(x=0)\right)}\right]^{q f_{d}}\left[v_{n} s^{\left(\delta_{d} @(x=-1)\right)}\right]^{1-q f_{d}}
\end{aligned}
$$




\subsection{Calculation and Plotting of Compound Derived Exponents, $q f_{d}$, $\exp _{d}$, and Derived $\delta_{d}$ Values}

Many of the fundamental constants are compound product/ratios of other fundamental constants. Therefore $t_{P}^{2}$ is a compound of four other entities. All of the possible valid force $\delta_{d}$ lines of these composite forces can be derived since they must represent sums and differences of the slopes and $y$ intercepts of the wk and EM lines, bwk, awk, and bem, as in the $H_{0 d} \delta$-line, Equations (19), (20). [14] These are the only possible valid values in a unified harmonic system, Figure 3, Figure 4. All of the compound values are derived.

$$
\begin{aligned}
& x_{\text {compound }}=\Sigma\left(q f_{\text {compound }}\right)-1=\Sigma\left(1 \pm \frac{1}{n_{\text {compound }}}\right)-1 \text { for } n=1 \text { to } 12 \text { and products } \\
& y_{\text {compound }}=\delta_{\text {compound }}=\left(\text { slope }_{\delta \text { line_compound }}\right)\left(x_{\text {compound }}\right)+y_{\delta \text { line_compound } @ x=0} \\
& y_{\text {compound }}=\delta_{\text {compound }}=\left(\Sigma_{\delta \text { compound } @ x=0}-\Sigma_{\delta \text { compound } @ x=-1}\right)\left(x_{\text {compound }}\right)+\Sigma_{\delta \text { compound } @ x=0} \\
& y_{\text {compound }}=\delta_{\text {compound }}=\left(\Sigma q f_{\text {compound }}\right)\left(\Sigma \delta_{\text {compound } @ x=0}\right)+\left(1-q f_{\text {compound }}\right)\left(\Sigma \delta_{\text {compound } @ x=-1}\right) \\
& y_{\text {compound }}=\delta_{\text {compound }}=f\left[\left(\Sigma q f_{\text {compound }}-1\right)\left(\Sigma_{\text {compound }} \text { awk, bwk, bem }\right)+\left(\Sigma_{\text {compound }} \text { awk, bwk, bem }\right)\right]
\end{aligned}
$$

The compound values are plotted at their $q f_{d}-1 \mathrm{X}$-axis values and their $\delta_{d} \mathrm{Y}$-axis values identical to the other single physical value constants, Figure 4.

\subsection{Calculation of Known and Derived Proportionality Constants}

Equation (21) is the proportionality constant, known or derived $k_{k}$ or $k_{d}$, multiplied with $\left(v_{n^{0}}\right) s$ that is associated with another fundamental constant. Equation (22) is the basic form of this relationship used in the proportionality constant derivations.

$$
\begin{aligned}
& v_{d}=k_{d}\left(v_{n^{0}}\right) s, \quad v_{k}=k_{k}\left(v_{n^{0}}\right) \text { or } k_{d}=\frac{v_{d} \mathrm{~Hz}}{v_{n^{0}} \mathrm{~Hz}}, k_{k}=\frac{v_{k} \mathrm{~Hz}}{v_{n^{0}} \mathrm{~Hz}} \\
& k_{d}=\frac{v_{d} \mathrm{~Hz}}{v_{n^{0}} \mathrm{~Hz}}=v_{n} s^{\left(q f_{d}\right)+\left(q f_{d}-1\right)\left(\Sigma_{d} \text { awk, bwk,bem }\right)+\Sigma_{d} \text { awk,bwk, bem }}
\end{aligned}
$$

\subsection{Calculation and Plotting of the Known Frequency Equivalent $t_{P k}^{2}, \exp _{k}, \delta_{k}$, and $2 d$ Point}

The known experimental h-bar $t_{p}$ is $5.39106(32) \times 10^{-44} \mathrm{~s}$, relative error $6 \times 10^{-5}$. The known experimental non h-bar $t_{p}$ is $1.35134(81) \times 10^{-43} \mathrm{~s}$, Table 1 . The $t_{p}^{2} \mathrm{~s}^{2}$ non h-bar is $1.82611(11) \times 10^{-86} \mathrm{~s}^{2}$. The known mean experimental non h-bar $\exp _{\mathrm{k}}$ is $-3.670879(12)$. The known experimental non h-bar $\delta_{k}$ is $-1.37371(1) \times$ $10^{-2}$. The $q f$ of $t_{P}^{2}$ is the sum of $-1,-1,-6 / 7,-4 / 5$ or $-128 / 35$ or -3.657142857142 . The $t_{P}^{2}$ point is plotted at $x=-128 / 35-1$, which equals $-163 / 35$.

\subsection{Calculation and Plotting of the Derived Frequency Equivalent $t_{P d}^{2}, \exp _{d}, \delta_{d}$ Point, $\delta_{d}$ Line}

The harmonic neutron hypothesis has derived a high accuracy Planck time $t_{P}$. [9] The $q f$ for these four entities defining $t_{P}^{2}$ are -1 , the gravitation binding energy of the electron -1 , negative qf proton, $-6 / 7$, negative $q f$ electron; -4/5; negative of Bohr radius. The sum equals $-128 / 35$. This is plotted at the $x$-value of $-163 / 35$, $-128 / 35-1$.

The derived $t_{P}^{2}$ compound $\delta_{d}$ line has a slope of awk - bwk - bem, and a $y$ intercept of -bwk - bem at $x$ equals 0 , and $y$ intercept of -awk at $x$ equals -1 , Equations (23)-(25), Figure 4. [9] The derived $\exp _{d}$ is $-3.670879366(1)$, the $\delta_{d}$ is $-1.3736509(1) \times 10^{-2}$ and the derive $v_{t P d}^{2}$ is $1.82617126(91) \times 10^{-86} \mathrm{~s}^{2}$ relative 
errors of $5 \times 10^{-8}$. The derived no h-bar $n_{t P d}$ equals $1.35135904(68) \times 10^{43}$. The equivalent $v_{P d}$ h-bar value is $5.39114257(27) \times 10^{-44} \mathrm{~s}$. All these values are within the known experimental values.

$$
\begin{aligned}
& \delta_{t_{P}^{2} d}=-\mathrm{awk}+\left(-\frac{128}{35}\right)(\mathrm{awk}-\mathrm{bwk}-\mathrm{bem})=-1.3736509(1) \times 10^{-2} \\
& \exp _{t_{P}^{2} d}=-\frac{128}{35}-\mathrm{awk}+\left(-\frac{128}{35}\right)(\mathrm{awk}-\mathrm{bwk}-\mathrm{bem})=-3.670879366(1) \\
& v_{t_{P}^{2} d}=\left[\left(v_{n^{0}}\right) s\right]^{-\frac{128}{35} \text {-awk }+\left(-\frac{128}{35}\right)(\text { awk-bwk-bem })}=1.82617126(91) \times 10^{-86} \mathrm{~s}^{2}
\end{aligned}
$$

\subsection{The Known Experimental Velocities, $H_{0} \mathrm{~S}^{-1}$, $\exp _{k}$, and $\delta_{k}$ Ranges}

The known experimental velocities at $\mathrm{Mpc}^{-1}$ are $76.9^{+3.9}{ }_{-3.4}{ }^{+10.0}{ }_{-8.0} \mathrm{~km} \cdot \mathrm{s}^{-1} \cdot \mathrm{Mpc}^{-1}, 74.3 \pm 2.1 \mathrm{~km} \cdot \mathrm{s}^{-1} \cdot \mathrm{Mpc}^{-1}$, $69.32 \pm 0.80 \mathrm{~km} \cdot \mathrm{s}^{-1} \cdot \mathrm{Mpc}^{-1}, 67.3 \pm 1.2 \mathrm{~km} \cdot \mathrm{s}^{-1} \cdot \mathrm{Mpc}^{-1}$, Table 1 . These are converted to standard $\mathrm{s}^{-1} H_{0}$ values by dividing the velocity by one Mpc, $3.085677580 \times 10^{22}$ meters. These value are $2.49(12) \times 10^{-18} \mathrm{~s}^{-1}, 2.408(67)$ $\times 10^{-18} \mathrm{~s}^{-1}, 2.24(2) \times 10^{-18} \mathrm{~s}^{-1}$, and 2.18(4) $\times 10^{-18} \mathrm{~s}^{-1}$. Their respective exp $\mathrm{k}_{\mathrm{k}}$ are $-7.53(12) \times 10^{-1},-7.543(8) \times$ $10^{-1},-7.556(16) \times 10^{-1}$, and $-7.562(3) \times 10^{-1}$. The respective $\delta_{k}$ are: $-3.70(90) \times 10^{-3},-4.33(50) \times 10^{-3}$, $-5.62(20) \times 10^{-3}$, and lastly, $-6.17(32) \times 10^{-3}$.

\subsection{The Known Experimental Line Fit Values for $H_{0 k}$ and $t_{P k}^{2}, \exp _{k}, \delta_{k}$, Slope and $y_{k}$-Intercept at $x=0$}

A line fit of the known $t_{p}^{2}$ and $H_{0}$ data points was completed. The line fit $t_{P}^{2}$ and $H_{0}$ values are respectively $1.82611(11) \times 10^{-86} \mathrm{~s}^{2}$ and $2.33(11) \times 10^{-18} \mathrm{~s}^{-1}$. The slope is $3.021 \times 10^{-3}$, and the $y$-intercept at $x=0$ is $3.315 \times 10^{-4}$. The line fit $\exp _{k}$ and $\delta_{k}$ for $t_{P k}^{2}$ are $-3.670879(12)$, and $-1.37371(1) \times 10^{-2}$. This is compared to the derived values in Table 1, Figure 4. The derived and known values correlate well.

\subsection{Derivation of the $\exp _{d}, \delta_{d}$ for $q f$ Values of $-1 / 2,-3 / 4,-5 / 6$, and $-6 / 7$ on the $t_{P d}^{2} \delta_{d}$ Line}

The generalized $t_{P d}^{2} \delta_{d}$ line for any $q f$ value is Equations (26)-(28). This equation is used to derive the possible hypothesized negative value harmonic fractions of beta neutron decay, $-1 / 2,-3 / 4,-5 / 6,-7 / 8$. One of these is hypothesized be related to $H_{0}$.

$$
\begin{aligned}
& \delta_{\text {dgeneralized }}=-\mathrm{awk}+(q f)(\mathrm{awk}-\mathrm{bwk}-\mathrm{bem}) \\
& \exp _{d g e n e r a l i z e d}=q f-\mathrm{awk}+(q f)(\mathrm{awk}-\mathrm{bwk}-\mathrm{bem}) \\
& v_{\text {dgeneralized }}=\left(v_{n^{0}} s\right)^{q f-\mathrm{awk}+(q f)(\mathrm{awk}-\mathrm{bwk}-\mathrm{bem})}
\end{aligned}
$$

The derived $\delta$, exponents, and $\mathrm{s}^{-1}$ calculated values from the $t_{P d}^{2}$ line for the possible $q f s$ of $-1 / 2,-3 / 4$, $-5 / 6,-7 / 8$ are respectively for $-1 / 2:-4.4681966(3) \times 10^{-3},-5.0446820(13) \times 10^{-1}, 1.6498650(1) \times 10^{-12} \mathrm{~s}^{-1}$, for $-3 / 4$ : $-5.20211263(26) \times 10^{-3},-7.5520211(04) \times 10^{-1}, 2.2972668(2) \times 10^{-18} \mathrm{~s}^{-1}$, for $-5 / 6$ : $-5.4467515(3) \times$ $10^{-3},-8.3878008(4) \times 10^{-1}, 2.5652661(7) \times 10^{-20} \mathrm{~s}^{-1}$, for $-7 / 8$ : $-5.5690708(3) \times 10^{-3},-8.8056907(5) \times 10^{-1}$, 2.7107717(3) $\times 10^{-21} \mathrm{~s}^{-1}$, Figure 4. The $q f$ of $-3 / 4$ is most the associated $q f$ of $H_{0}$. The derived $\delta_{H_{0}}$ from this method is the intercept of the $t_{P}^{2} \delta_{d}$ line at an $x$ value of $-3 / 4-1$. This is the harmonic fraction $x$ location of $-3 / 4-1,-7 / 4$. The specific derivation of these factors for $H_{0}$ are shown in Equations (29)-(31).

$$
\begin{aligned}
& \delta_{H_{0}}=\left(-\mathrm{awk}-\frac{3}{4} \times(\mathrm{awk}-\mathrm{bwk}-\mathrm{bem})\right)=-5.20211263(26) \times 10^{-3} \\
& \exp _{H_{0} d}=-\frac{3}{4}+\left(-\mathrm{awk}-\frac{3}{4} \times(\mathrm{awk}-\mathrm{bwk}-\mathrm{bem})\right)=-7.5520211(04) \times 10^{-1}
\end{aligned}
$$




$$
H_{0 d}=\left(v_{n^{0}} S\right)^{\left(-\frac{3}{4}+\left(- \text { awk }-\frac{3}{4} \times(\text { awk-bwk-bem })\right)\right.}=2.2972668(2) \times 10^{-18}
$$

\subsection{Derived Hubble Time, Hubble Length}

The derived Hubble time is the inverse of $H_{0}, 4.3529990(17) \times 10^{17}$ seconds. There are $3.1556926 \times 10^{7}$ seconds per year. The derived Hubble time equals $13.7941161(13) \times 10^{9}$ years. The derived Hubble length is $13.7941161(13) \times 10^{9}$ light years. The reported experimental value is approximately $13.8 \times 10^{9}$ light years.

\subsection{Comparison of Known and Derived Proportionality Constants of $t_{P}^{2}, H_{0}$ and the Neutron}

The known proportionally constant of the ratio of $\delta_{k}$ line fit $H_{0}$ divided by $v_{n^{0}}$ is $1.02(15) \times 10^{-41}$. The derived value is $1.01118370(16) \times 10^{-41}$, Equation (32), Table 4 .

$$
k_{d H_{0} / v_{n^{0}}}=\frac{H_{0 d}}{v_{n^{0}}}=\left[\left(v_{n^{0}}\right) s\right]^{\left(-1-\left(-\frac{3}{4}+\left(- \text { awk }-\frac{3}{4} \times(\text { awk-bwk-bem })\right)\right)\right)}=1.01118370(16) \times 10^{-41}
$$

The known proportionally constant of the ratio from the $\delta_{k}$ line fit $t_{P}^{2}$ divided by $v_{n^{0}}$ is $8.03(40) \times 10^{-110}$. The derived value is $8.0382241(5) \times 10^{-110}$, Equation (33).

$$
k_{d t_{P}^{2} / v_{n^{0}}}=\frac{t_{P}^{2}}{v_{n^{0}}}=\left[\left(v_{n^{0}} s\right)\right]^{\left(-1-\frac{128}{35} \text {-awk- }-\frac{128}{35} \times(\text { awk-bwk-bem })\right)}=8.0382241(5) \times 10^{-110}
$$

The known proportionally constant of the ratio from the $\delta_{k}$ line fit $H_{0}$ divided by $t_{P}^{2}$ is $1.272(64) \times 10^{68}$. The derived $k_{d}$ value is $1.25796898(7) \times 10^{68}$, Equation (34).

$$
k_{d H_{0} / t_{P}^{2}}=\frac{H_{0}}{t_{P}^{2}}=\left[\left(v_{n^{0}} s\right)\right]^{\left(\frac{128}{35}-\frac{3}{4}+\left(\frac{128}{35}-\frac{3}{4}\right) \times(\text { awk-bwk-bem })\right)}=1.25796898(7) \times 10^{68}
$$

\section{Discussion}

A robust physics model that explains many of the mysteries of today remains elusive [18] [19]. A dominant unsolved problem is how to scale sub-atomic quantum, classical physics, and cosmologic phenomena simultaneously in a coherent mathematical and physical model. The HNH answers some of these questions, and actually derives accurate values of the physical cosmological and high energy constants that cannot be accurately experimentally measured [7]-[14].

Many critics of the HNH suspect that these findings are simply coincidence or numerology. The $\mathrm{HNH}$ is however a classic dimensionless physical system of the Buckingham Pi theorem type. The speed of light is finite, and constant within any setting. It is logical that the whole system should be based on a finite constant as well. The harmonic neutron hypothesis is highly restricted. Only three starting finite number sets are used. The derivations are not made directly from the original subatomic data, but from the unified scaling of the whole universal harmonic 2d plane, Figure 3, Figure 4. Therefore it is incorrect to interpret that $H_{0}$ was derived from a product ratio relationship of the four subatomic constants that would be utilized in a classic physics' method. It is impossible to manipulate the results since all of the components are fixed previously published natural units, and harmonic integer fractions. It is not possible to derive any value by manipulating the $n_{\text {ife }}$ value. An argument by analogy is that it is not possible to derive any wavelength from the Rydberg series by changing the $n_{1}$ and $n_{2}$ values since $R$ is a natural unit

The hypothesis logically states that related physical constants will naturally all fall on a single $\delta$ line since they are all scaled by the same proportionality constant. The derivation of $H_{0}$ from the $t_{P d}^{2} \delta_{d}$-line is support of the hypothesis that these two are related to the same proportionality constant, Equations (32)-(34). The proportionality constants of the neutron, $t_{p}^{2}$, and $H_{0}$ are accurately derived. This is viewed as impossible utilizing standard methods.

The typical interpretation of the $H_{0}$ is that it is not felt to be a true constant, but changes with other variables 
defining the nature of the cosmos. It has been described as the Hubble parameter. It is experimentally impossible to prove that the $H_{0}$ is actually changing from the present value. In the HNH $H_{0}$ is felt to constant, and is analogous to the free space constants of permeability, and permittivity. Interpretation of quantum systems using classical physics concepts is inaccurate and inappropriate. In the HNH the same is true for cosmology phenomena.

It is logical that the $H_{0}$ should be closely related to the gravitational force, and therefore $t_{p}^{2} . H_{0}$ is related to an expansive kinetic phenomenon, and so is the neutron beta decay process. $H_{0}$ and the beta decay $q f s$ have inverse signs, $3 / 4$ and $-3 / 4$, but identical harmonic fractions. The harmonic neutron hypothesis has shown multiple examples of this type of harmonic fraction sign symmetry with inverse sign relationships. The top quark is $1+1 / 10$ and the up quark is $1-1 / 10$. The Higgs boson is $1+1 / 11$, and the down quark is $1-1 / 11$. This is a non-coincidental relationship as seen with the Rydberg series and with Moseley's law, which in the exponential domain represent inverse exponents.

Perhaps the other $q f s-1 / 2,-5 / 6$, or $-7 / 8$ represent the properties of dark matter and energy. It is possible to accurately derive CMB peak spectral radiance from the same $t_{p}^{2}$ line using the partial fractions $1 / 2$ and $2 \times$ $-3 / 4,-3 / 2$. It is likely that dark matter and dark energy are related to the partial fraction $-1 / 2$. The properties of black holes can also be interrogated with the HNH without limitations of singularities since the Schwarzschild radius relationship can be evaluated using $t_{p}^{2} \mathrm{~s}^{2}$. The derived $t_{P}^{2}$ can be used to derive the masses and dimensions of black holes. Also the $\mathrm{HNH}$ has no singularities since the number $V_{f}$ is finite, and also represents the maximum Lorentz factor.

The HNH also explains the precise logical origin of $H_{0}$ and unification with other fundamental constants including the neutron, hydrogen, neutrinos, $t_{p}$, G, and the kinetic energy lost in the beta decay process. The $+3 / 4$ $q f$ is associated with kinetic energy lost during neutron beta decay. It is also associated with the muon antineutrino, as yet unpublished data. The expectation values of the neutrinos all fall on the wk line. The expectation value of the muon neutrino is related to the $q f$ sum of $3 / 4$ and $1 / 10$ for the up quark on the wk line. The expectation value of the electron neutrino is related to the $q f$ sum of $1 / 2$ and $1 / 7$ for the electron on the wk line. The expectation value of the Tau neutrino is related to the $q f$ sum of 5/6 and 1/10, and for the up quark on the wk line.

\section{Conclusion}

$H_{0}$ can be derived from four finite integer natural units and $N_{12}$. $H_{0}$ is logically related by harmonic fractions to the beta decay kinetic energy based on a common harmonic fraction, 3/4, but with opposite sign. The experimental Planck time and $H_{0}$ data power law data is closely linked to the predicted data. The derived $H_{0}$ can be evaluated in the future to see if this is an accurate prediction. Derivation of accurate coupling constants of the neutron with $t_{p}^{2}$ and $H_{0}$ has never been achieved before so this is a significant result.

\section{Acknowledgements}

I would like to thank Tom Budinger Ph.D. for his sage advice, and help. I would also like to thank Richard White MD for his support of this work.

\section{References}

[1] Ade, P.A.R., Aghanim, N., Alves, M.I.R., et al. (2014) Astronomy \& Astrophysics, 571, Article ID: A1.

[2] Bonamente, M., Joy, M.K., Laroque, S.J., Carlstrom, J.E., Reese, E.D. and Dawson, K.S. (2006) The Astrophysical Journal, 647, 25-54. http://dx.doi.org/10.1086/505291

[3] Freedman, W.L. and Madore, B.F. (2010) Annual Review of Astronomy and Astrophysics, 48, 673-710. http://dx.doi.org/10.1146/annurev-astro-082708-101829

[4] Bennett, C.L., Larson, D., Weiland, J.L., Jarosik, N., Hinshaw, G., Odegard, N., Smith, R.S., Hill, K.M., Gold, B., et al. (2013) The Astrophysical Journal Supplement Series, 208, 19.

[5] Freedman, W.L., Madore, B.F., Scowcroft, V., Burns, C., Monson, A., Persson, S.E., Seibert, M. and Rigby, J. (2012) The Astrophysical Journal, 758, 24.

[6] Ade, P.A.R., Aghanim, N., Armitage-Caplan, C., Arnaud, M., Ashdown, M., Atrio-Barandela, F., et al. (2014) Astronomy \& Astrophysics, 571, Article ID: A16.

[7] Chakeres, D.W. (2009) Particle Physics Insights, 2, 1-20. 
[8] Chakeres, D.W. (2011) Particle Physics Insights, 4, 19-23. http://dx.doi.org/10.4137/PPI.S7961

[9] Chakeres, D.W. (2011) Particle Physics Insights, 4, 25-31. http://dx.doi.org/10.4137/PPI.S8241

[10] Chakeres, D.W. (2013) Particle Physics Insights, 6, 1-7. http://dx.doi.org/10.4137/PPI.S12390

[11] Chakeres, D.W. (2011) Particle Physics Insights, 4, 33-38. http://dx.doi.org/10.4137/PPI.S8269

[12] Chakeres, D.W. (2012) Bulletin of the American Physical Society, 57.

[13] Chakeres, D.W. (2006) The Imaginary Number Neutron Symphony. US Copyright, TXu1-295-777/2006-09-15.

[14] Chakeres, D.W. (2014) Journal of Modern Physics, 5, 1670-1683. http://dx.doi.org/10.4236/jmp.2014.516167

[15] Lauterbur, P.C. (1973) Nature, 242, 190-191. http://dx.doi.org/10.1038/242190a0

[16] Ljunggren, S. (1983) Journal of Magnetic Resonance, 54, 338-343. http://dx.doi.org/10.1016/0022-2364(83)90060-4

[17] Cajori, F. (1909) A History of the Logarithmic Slide Rule and Allied Instruments. The Engineering News Publishing Company, New York.

[18] Ng, Y.J., Christiansen, W.A. and van Dam, H. (2003) The Astrophysical Journal, 591, L87-L89. http://dx.doi.org/10.1086/377121

[19] Lykken, J. and Spiropulu, M. (2014) Scientific American, 310, 34-39. http://dx.doi.org/10.1038/scientificamerican0514-34 